\title{
Le beurre et l'argent du beurre. Intérêt et limites du concept "genre" dans les études préalables aux projets de développement
}

\section{Laurence Boutinot}

\author{
(2) OpenEdition \\ Journals \\ Édition électronique \\ URL : http://journals.openedition.org/apad/251 \\ DOI : 10.4000/apad.251 \\ ISSN : 1950-6929 \\ Éditeur \\ LIT Verlag
}

Édition imprimée

Date de publication : 20 décembre 2000

\section{Référence électronique}

Laurence Boutinot, «Le beurre et l'argent du beurre. Intérêt et limites du concept "genre" dans les études préalables aux projets de développement », Bulletin de l'APAD [En ligne], 20 | 2000, mis en ligne le 03 avril 2006, consulté le 08 septembre 2020. URL : http://journals.openedition.org/apad/251 DOI : https://doi.org/10.4000/apad.251

Ce document a été généré automatiquement le 8 septembre 2020.

Bulletin de I'APAD 


\title{
Le beurre et l'argent du beurre. Intérêt et limites du concept "genre" dans les études préalables aux projets de développement
}

\author{
Laurence Boutinot
}

"Entre femme et femme c'est comme entre vache
et vache. Lorsque l'une voit où pousse la bonne
herbe, elle n'appelle pas l'autre. "
Proverbe peul.

Introduction

1 Les femmes sont présentes à tous les maillons de la filière économique du lait (laban) au Tchad. Productrices ou commerçantes, elles y occupent une position doublement légitime, comme détentrices d'un savoir technique (transformation du lait) et comme héritières d'un statut économique et social.

2 Cette filière du lait est actuellement en mutation. L'accession d'Idriss Déby au pouvoir, les politiques d'aide à la reconstruction économique du pays imposées par la Banque mondiale et le FMI, la dévaluation du franc CFA en 1994, on marqué la dernière décennie. La paix relative a entraîné une démobilisation massive et il n'est donc pas surprenant d'observer une réorganisation des activités économiques. L'implication de nouveaux acteurs masculins, éleveurs mais surtout commerçants, modifie depuis quelques années les rapports de force dans la filière du lait et, en particulier, les rapports entre les hommes et les femmes.

3 Cet article nous pousse à une réflexion sur le concept de genre ${ }^{1}$, sur son intérêt et ses limites dans le cadre de la programmation d'actions de développement. L'étude s'appuie sur une mission réalisée au Tchad en 1998 sans le cadre de la mise en place d'un projet visant l'amélioration de l'approvisionnement laitier de la ville de Ndjaména. Je souhaite mettre en lumière les contradictions, voire les apories, que l'utilisation d'un concept imprécis produit au niveau des pratiques sociales comme à 
celui de leur analyse. Trois axes de démonstration seront privilégiés, touchant chacun à la fois à une étape de l'étude concernée (présupposés, contexte et enjeux) et à l'approche théorique qu'elle représente (femme, hiérarchie et limites de l'analyse selon le genre).

Récurrence de la problématique laitière au Tchad

L'élevage est, au Tchad, la seconde source de richesse (16 \% du PIB) après le coton. Le bétail représente $30 \%$ des échanges et l'économie pastorale concerne $40 \%$ de la population active ${ }^{2}$. Les produits laitiers locaux couvrent environ entre $51 \%$ et $65 \%$ des besoins de la consommation urbaine (Koussou Mian Ondanang, 1998), l'importation couvrant le solde de la demande. L'approvisionnement de la capitale tchadienne en produits de l'élevage est, depuis l'époque coloniale, un thème récurrent qui s'avère d'autant plus pertinent en ce moment où la croissance démographique urbaine est importante (700 000 habitants ; 4,5\% par an), supérieure à celle de la population rurale $(2,7 \%$ par an) et plus encore à celle du cheptel bovin $(2,5 \%)$. Cette dynamique démographique pourrait rendre la ville de Ndjaména de plus en plus dépendante des produits importés, comme le sont de nombreuses capitales de l'Afrique de l'Ouest.

Le Ministère de l'Elevage tchadien, sollicité par un député concerné par les productions animales de sa région, a demandé l'intervention d'un bailleur de fonds français en vue de restructurer la filière économique. Comme chacun sait, la mise en place d'un projet requiert la réalisation d'études préalables : intérêt économique du projet, faisabilité et premières recommandations de mise en place. Une mission principale agrémentée, si besoin est, d'études complémentaires permet de réaliser ces études. C'est ainsi que, suite à une première mission d'identification de la filière, une étude selon le "genre" (gender approach) a été demandée dans le cadre des études complémentaires nécessaires à établir la faisabilité du projet.

6 Un des objectifs du projet est de contribuer à une structuration rentable de J'économie pastorale en effectuant des rationalisations. Dans de nombreuses opérations de développement en Afrique, la rentabilisation marchande d'une production agricole (riz), fruitière (karité), forestière (gomme arabique) ou pastorale, a pour conséquence que les hommes s'emparent du marché (Bisilliat 1997: 38). Le projet ici étudié n'y échappe pas. C'est le cas ici du produit laitier à l'état frais (hlib) qui attire un nombre grandissant d'hommes, éleveurs ou simplement commerçants. Ces nouvelles pratiques masculines, dans un domaine économique jusqu'alors féminin, vont modifier les pratiques des femmes productrices et jusqu'au cœur des maisons et des couples, et aussi les pratiques des femmes commerçantes collectrices de lait caillé (rouaba; lait fermenté écrémé) dont la part de marché se rétrécit au profit d'une collecte de lait frais par les hommes. Elles ne peuvent entrer en compétition avec ces concurrents plus rapides (ils disposent de mobylettes) et mieux organisés.

Concept "genre" ou conception de "la femme" ?

7 Le projet de développement en question entend se démarquer d'expériences avortées au Tchad ${ }^{3}$ mais ses objectifs n'en restent pas moins ambitieux, puisque le but est d'intensifier la production laitière et de rentabiliser ses formes marchandes.

Mission d'identification : contexte d'une étude de projet

La première mission d'identification réalisée par des chercheurs vétérinaires d'un Institut national tchadien s'est déroulée en mai 1997. L'impact de cette mission d'identification est perceptible, dès l'année suivante. Déjà, un nombre grandissant d'hommes commerçants et éleveurs a modifié la filière en se mobilisant pour la collecte 
du lait frais. Notons que la préfecture du Chari-Baguirmi est encadrée par divers projets d'organisation paysanne et pastorale ${ }^{4}$, ce qui a familiarisé les acteurs locaux, surtout masculins, avec les démarches de sollicitation.

9 La mission d'identification est une approche technique et économique de la production laitière. Elle met en évidence les limites des possibilités d'approvisionnement laitier de la ville de Ndjaména, notamment en saison sèche ${ }^{5}$. Afin de ne pas voir cette situation devenir critique, elle recommande deux séries d'actions : d'une part, une adaptation des systèmes de production et de transformation, d'autre part, une extension de la zone de collecte. Ce deuxième point ne sera pas traité ici puisqu'il concerne un volet du projet destiné à désenclaver un département situé hors de l'étude traitée.

évequees de l'insuffisance de la production laitière de la zone périphérique de Ndjaména sont la pénurie des pâturages, la déficience des vétérinaires et l'approvisionnement défectueux en médicaments ${ }^{6}$. Un projet de promotion de l'élevage péri urbain s'appuyant sur des groupements d'éleveurs (Souvenir Zafindrajaona 1997 : 61) semble adéquat. Il préconise des pratiques nouvelles de gestion des troupeaux consistant à les diviser en deux lots différents : un lot productif de vaches en lactation fixé au village d'origine des éleveurs, d'une part, un lot non productif (mâles, jeunes, vaches taries et loin du terme pour les vaches pleines), mis en transhumance.

11 Cette division du bétail modifierait la répartition des tâches entre les membres de l'exploitation mais permettrait, en revanche, de réduire les risques de surpâturages autour de Ndjaména, le lot sédentarisé étant alimenté de tourteaux de coton. L'augmentation de la production laitière et la vente de lait frais, plus rentable, devraient, selon les calculs ${ }^{7}$, compenser le surcoût des compléments alimentaires. Les recommandations d'ordre sanitaire concernent l'appui aux vétérinaires et viseraient surtout une meilleure organisation (GIE). Seule l'éventualité d'une production de fromage pour recycler les surplus saisonniers du lait s'adresse aux groupements de femmes. La suggestion s'accompagne de la mention d'une association possible avec l'entreprise SOMAFA ${ }^{8}$, spécialiste de la transformation des surplus laitiers en beurre et fromage.

12 Les recommandations de la mission d'identification éclairent le contexte et ses présupposés androcentriques dont l'essentiel participe d'une vision purement économique du projet. Les travaux d'enquêtes, réalisés exclusivement par des chercheurs vétérinaires et économistes masculins, s'inscrivent dans le cadre de la demande du ministère de l'Elevage et s'adressent aux hommes du monde pastoral. De plus, les recommandations formulées touchent à la rentabilité et à l'amélioration de la gestion des troupeaux dans les circuits marchands de vente du lait à l'état frais où. nous l'avons vu, les hommes sont mieux outillés. De même en est-il de la complémentation alimentaire des animaux dont le coût pourrait être compensé par la vente du lait frais. Le manque à gagner des femmes, et en particulier des femmes collectrices de lait caillé, n'est jamais évoqué. Enfin, la fabrication de fromage, éventuellement laissée aux femmes, requiert" l'adhésion volontaire des femmes et leur participation financière" et nécessite une formation technique et d'encadrement (animatrices et productrices de fromage). Quoi qu'il en soit, une association avec la SOMAFA rendrait plus improbable encore la participation des femmes d'éleveurs.

13 L'étude sur le "genre" (gender approach) a été demandée en complément de la première mission identificatoire. Ce sont, du reste, les bailleurs de fonds, dont la démarche 
s'inscrit dans la ligne recommandée par l'OCDE, qui ont demandé cette étude, et non le ministère des Affaires sociales et de la Promotion féminine du Tchad. Cette étape va s'inscrire "tout naturellement" dans la conception première et se diriger spécifiquement vers les femmes : la demande d'analyse des rapports socio-économiques de genre y échappe. Les termes de référence de l'étude consistaient, en effet, à répondre aux questions suivantes : comment s'organise la division sexuelle du travail dans la production et la commercialisation du lait autour de Ndjaména? Quelles sont les actions susceptibles d'être menées auprès des femmes dans le cadre de la structuration de la filière du lait? Faut-il créer de nouvelles structures, utiliser celles existantes ou laisser dans l'informel les activités qui s'y déploient? Les prémisses du projet reflètent le rapport androcentrique des concepteurs à la réalité.

Logique développementaliste et présupposés androcentriques

L'invisibilité du travail productif des femmes s'inscrit dans la vision occidentale du monde à la fois ethnocentrique et androcentrique. Les concepteurs pensent le projet selon le modèle de l'évolution de la production laitière à l'époque de l'industrialisation européenne du XIX et envisagent le travail des femmes rurales comme partie domestique d'un travail conjugal. La sous-filière de production laitière coutumièrement féminine évoque pour eux, les bons souvenirs des systèmes d'élevage du Moyen Age français où le lait, un "sous-produit", est traité, par les femmes, dans la laiterie : son traitement est "une activité féminine relevant de l'espace domestique" (Vatin 1990 : 16). Les "modèles idéologiques dominants qui constituent l'agriculture en activité patrimoniale et le travail de l'agricultrice en "travail d'épouse" élargi" (Lagrave 1987 : 26) contaminent le monde rural en général et l'élevage en particulier. Dans une telle perspective, l'étude selon le genre se retranche "tout naturellement" dans la formulation de recommandations compensatrices pour les femmes. L'étude sur le genre apparaît comme complémentaire et secondaire. Au moment où elle est lancée, il ne s'agit plus que de saisir ce que sera la position des femmes dans un contexte économique déjà modifié en faveur d'une dynamique masculine. Malgré les recommandations des organisations internationales, les femmes ne sont toujours pas associées, au même titre que les hommes, aux conceptions, aux décisions et aux élaborations de projets. "Le poids des mentalités", euphémisme sexuellement neutre (ibid. : 18), aboutit à un rejet d'une étude prenant en compte les rapports de genre, tant dans l'équipe du projet que dans les différents réseaux d'acteurs où le projet vise son insertion. La puissance des présupposés androcentriques ne se dévoile jamais aussi bien que lorsque la femme est prise comme objet de l'étude, quand bien même il s'agirait d'en vanter les qualités ou bien d'en améliorer le sort. Une première rupture consiste alors à la considérer comme sujet de l'étude. "Dans nombre de sociétés, les femmes sont des pions, mais on ne peut pleinement le démontrer qu'en les prenant comme sujets de l'analyse..." (Mathieu 1991a : 16)... ce que l'étude des rapports de genre permettrait.

Division sexuelle du travail ou "hiérarchisation socio-sexuée" du travail ?

Les femmes du milieu agro-pastoral tchadien sont peu connues et il est donc commode de les considérer formant une entité homogène. Les résultats de notre enquête auprès des actrices et des acteurs de la filière lait nous montrent l'hétérogénéité du milieu des producteurs et des productrices, des commerçantes et des commerçants. Les intérêts divergent, aussi bien que les pratiques marchandes et que les unités de production. L'organisation du travail varie, mais également la répartition des avoirs et les modes de redistribution des biens produits diffèrent. Le bétail et son produit laitier ne revêtent 
pas les mêmes significations sociales et économiques selon les milieux. L'intérêt que l'on porte à mettre en évidence les variations de l'organisation du travail dans les différents modes de production tient moins de la description de la division sexuelle du travail que d'une mise à jour de la "hiérarchisation socio-sexuée" (Mathieu 1991a) du travail. Cette dernière est directement liée à la signification et la mise en valeur économique des produits de l'élevage.

Un monde pastoral non homogène

Parmi les productrices et les producteurs, nous avons rencontré ${ }^{9}$ principalement trois groupes ethniques: les Arabes du Chari-Baguirmi (ou Arabes Shuwa), les Arabes du Centre Tchad et les Peuls. La grande majorité des éleveurs de la région s'inscrit dans la catégorie des pasteurs cultivateurs ${ }^{10}$ sédentarisés ou, pour ce qui concerne les Peuls, passés à l'agriculture d'appoint. Quelques éleveurs "purs" se retrouvent chez certains transhumants et surtout chez les nomades du Centre Tchad. Notre échantillon comprend des sédentaires, des nomades et des transhumants que l'habitat distingue, allant de la case fixe au campement mobile. Les Arabes du Chari-Baguirmi se distinguent des autres groupes, réalisant un équilibre agro-pastoral propre aux populations du Tchad (Bonfiglioli 1981) dans la familiarité avec le monde urbain de la capitale.

Dans toutes les catégories, les femmes propriétaires de bovins et/ou de chamelles sont des femmes généralement âgées ${ }^{11}$. Les femmes d'éleveurs, elles mêmes non propriétaires de bétail sont, en général, des femmes arabes sédentaires du Chari-Baguirmi ou des femmes jeunes. Celles qui ne possèdent que de petits ruminants sont aussi des sédentaires, hormis une jeune femme Peule divorcée.

Les femmes sédentaires se procurent du bétail grâce à la thésaurisation de leurs gains comme travailleuses agricoles ${ }^{12}$. Les femmes transhumantes ont pu, elles aussi, acheter du bétail mais $20 \%$ seulement de leur troupeau a été acheté, le reste, comme c'est le cas pour la totalité des nomades, provenant de dons, dot, douaire et/ou héritage et, bien entendu, du croît du bétail. Le prêt de bétail est observé chez les transhumants et chez les nomades (Dupire 1970) mais il est en voie de disparition en milieu sédentaire (Bonfiglioli 1990).

Ces distinctions sont importantes. En effet, le bétail hérité et son croît sont la propriété personnelle et inaliénable des femmes. Les bêtes de dot sont propriété de la femme, mais, en cas de séparation des conjoints, la propriété sera négociée. Les bêtes acquises autrement restent souvent, en cas de séparation, propriété de l'homme ou des enfants.

Les hommes (mari et fils chez les transhumants et nomades, et raay ou berger rémunéré plus fréquemment chez les sédentaires) se chargent de l'entretien du bétail (abreuvement et pacage). Les femmes abreuvent les animaux restés au village ou au campement (vaches allaitantes, animaux malades) au moment où elles se procurent l'eau de la famille. Chez les nomades et les transhumants, la traite des vaches est attribuée aux femmes le matin et le soir. Par contre, chez les sédentaires, la traite semble s'effectuer de manière plus indifférenciée selon les disponibilités de chacun.

21 Pour un tiers des femmes rencontrées (sédentaires), les revenus laitiers proviennent de la vente du lait frais; pour les deux autres tiers, ils proviennent de la vente du lait caillé.

Le lait caillé est vendu 150 FCFA le litre et le lait frais 250 FCFA le litre, en saison sèche. Ils sont respectivement de 50 FCFA et de 100 FCFA moins chers en saison pluvieuse. En 
outre, ces prix varient encore selon l'éloignement de la capitale ${ }^{13}$. Le prix du lait caillé est plus variable car le marché est moins homogène et les unités de mesure diffèrent. $\mathrm{Au}$ détail, le lait caillé est acheté au cob. Un coro $=2,5 \operatorname{cob}^{14}$. La vente du lait caillé est une pratique ancienne et populaire. Le lait caillé est l'objet de spéculations et entre dans des pratiques d'échange de produits locaux entre sédentaires et nomades, essentiellement des échanges de lait contre des céréales.

Les revenus moyens provenant du lait frais (hlib) sont plus intéressants ${ }^{15}$ (entre 1300 FCFA par jour en saison sèche et 1570 FCFA en saison des pluies) que ceux provenant du lait caillé (rouaba) (entre 750 FCFA en saison sèche et 990 FCFA en saison des pluies). Pourtant, certaines femmes, notamment chez les Peuls, refusent de vendre le lait autrement que caillé. En effet, le lait frais est pur don de la nature, empreint d'un caractère sacré ${ }^{16}$. Réservé à la consommation et au partage, il ne saurait faire l'objet de commerce. De plus, le lait frais peut être vendu "entier" pour la transformation en yaourt (rayib) et pour sa consommation en ville. Le prélèvement de la crème et sa transformation en beurre n'est, dès lors, plus possible. Or, les revenus provenant du beurre (zibne) sont également importants. Ce beurre, généralement liquide, (hormis chez les femmes Peules où il est solide) est assez régulièrement fabriqué à raison d'une moyenne d'un litre par semaine et par productrice de lait caillé. En saison sèche, le litre se vend entre 1650 FCFA à Ndjaména et 1500 FCFA sur les marchés périurbains. En saison pluvieuse, le litre se vend 1000 FCFA. Ces chiffres sont une construction moyenne, puisque le beurre provient des invendus de lait caillé obtenu après un chauffage et une clarification suffisante. Cette fabrication permet" un stockage spéculatif" de plusieurs mois (Bourzat et al. 1998).

Il est intéressant de distinguer les revenus des femmes d'éleveurs selon leur mode d'organisation sociale. En effet, les femmes transhumantes sont celles dont la production, et donc les ventes, sont les moins importantes. En revanche, les femmes sédentaires sont bien représentées tant pour les revenus du lait frais que pour ceux du lait caillé, en saison sèche comme en saison pluvieuse. Enfin, les revenus des femmes nomades proviennent majoritairement du lait caillé mis sur le marché et sont plus élevés en saison sèche, lorsque les femmes se trouvent à proximité de la capitale.

La gestion des revenus du lait au sein des ménages diffère, elle aussi, selon les modes de vie. Il nous est apparu clairement que les "caisses à part", ou budgets des femmes séparés de ceux des maris, sont le fait de la majorité des femmes sédentaires alors que dans la catégorie des femmes transhumantes et nomades, les budgets sont le plus souvent, voire toujours, mis en commun, sauf en cas de polygamie. Ces pratiques dénotent une plus grande solidarité entre les époux nomades dont le mode de vie est plus précaire et où les déséquilibres familiaux et économiques sont plus difficiles à surmonter.

Que les budgets soient séparés ou communs, la majorité des revenus laitiers paie le mil et les autres céréales avant de servir à la satisfaction des "petits besoins" des femmes. Toutes les femmes transhumantes et nomades nous ont répondu que le mari vendait un animal pour acheter le mil seulement "quand l'argent du lait est fini". Pourtant ce sont ces femmes, en particulier les Peules, que l'argent du lait caillé fait rêver à des parures.

27 Ni les éleveurs ni leurs femmes ne forment, on le voit, un groupe homogène et, de plus, les différents modes de vie s'expriment dans un rapport particulier à la production laitière. Le statut des femmes, un statut que les revenus laitiers influencent, varie jusque dans l'organisation du travail domestique. Dans cette sphère, l'organisation du 
travail matérialise les rapports sociaux, notamment la marge d'autonomie relative des femmes.

Les femmes nomades, plus dépendantes que les autres du produit laitier pour l'alimentation de la famille et pour les revenus monétaires, sont aussi les moins autonomes. Les revenus provenant de la vente de lait sont les plus élevés pour les femmes sédentaires auxquelles ils donnent aussi une plus grande liberté dans l'acquisition individuelle de bien "urbains". Cette situation doit être mise dans le contexte d'une instabilité matrimoniale plus grande qui incite ou force les femmes à se protéger d'une trop grande dépendance des hommes. En effet, la sédentarisation des éleveurs va de pair avec "une implication plus grande des femmes dans le travail productif. (...) Elle tend à constituer des unités familiales plus larges, à renforcer le pouvoir d'un chef de famille unique et à retarder la constitution d'unités familiales autonomes, économiquement suffisantes" (Bonfiglioli 1990: 260). Les femmes transhumantes disposent de moins de revenus, de moins d'autonomie, et souffrent d'une charge de travail plus lourde. Elles semblent, contrairement à ce que certains auteurs ont pu montrer pour les hommes (Bonfiglioli 1990), contraintes dans un mode de vie qui cumule les risques de la précarité (accès à la terre non sécurisé, coût des bergers pendant la saison des pluies) sans préserver des difficultés propres au mode de vie nomade et au mode de vie sédentaire.

Les enjeux du projet et l'approche "genre" en termes de hiérarchisation du travail

Passant à une analyse sociologique, nous avons rencontré six groupements de femmes de la sous-préfecture de Ndjaména rurale, tous encadrés par la direction de l'Organisation pastorale. Peu d'activités y ont été concrétisées dans ces groupements récemment formés et institutionnellement quasi virtuels. Sur les six groupements visités, deux paraissent cependant plus dynamiques que les autres. Les quatre autres semblent être à l'étape des souhaits et surtout des demandes encore illusoires de l'assistance proprement dite. Les deux groupements les plus dynamiques ne sont pas les plus anciens mais sont les lieux où les maris des femmes productrices, également éleveurs et commerçants, sont les nouveaux collecteurs de lait frais à mobylette. Pour les membres de ces groupements, la production laitière s'ajoute aux activités agricoles ou artisanales qu'ils partagent avec les autres groupements.

Les femmes considèrent les revenus du lait comme "trop sérieux pour être mis en commun" et la filière s'intègre donc difficilement dans les actions du groupement. Les femmes nourrissent leur famille avec ces revenus et ne veulent pas les "risquer" dans une entreprise collective. Ces revenus sont individuels mais ceci n'empêche pas que les femmes soient à la recherche d'actions valorisant cet avoir. L'élevage leur paraît le contexte approprié. Certaines femmes, lors des réunions, ont résumé les actions que chacun des groupements avait envisagé autour de la commercialisation laitière. Pour les unes, le groupement pouvait être un intermédiaire supplémentaire entre les productrices et les collecteurs motorisés. Pour les autres, le groupement pouvait organiser une collecte indépendante et en parallèle, éventuellement concurrente de celle des maris collecteurs.

31 Ce niveau d'analyse collectif porte, de façon compréhensible, sur les femmes sédentaires. Celles-ci ont pressenti les enjeux du projet et l'intérêt de se réunir en groupement. Certes, les groupements féminins sont souvent restés vagues en comparaison avec les groupements d'hommes organisés pour des actions concrètes, mais la demande des pouvoirs publics à leur endroit était elle-même désincarnée. Dans 
les groupements de femmes, se produit une prise de conscience de l'importance des revenus laitiers et des convoitises qui pourraient, à terme, entamer la relative liberté d'utilisation dont elles jouissaient. Les femmes ne peuvent être actives au sein du groupement que si les hommes, dont l'autorisation est nécessaire, y trouvent leur intérêt. Elles envisagent donc l'utilisation d'une part de leurs revenus mis en commun dans le groupement pour l'achat d'une partie des compléments alimentaires pour le bétail, jusque là entièrement à charge des hommes. Ce qui allait de soi dans le contexte des pratiques anciennes redeviendrait, à travers l'organisation rationnelle des actions communautaires, un échange marchand au sein des ménages ruraux. Cette concession peut être vue comme un glissement du pouvoir masculin dans la sphère féminine, puisque les femmes ne bénéficieraient plus de la liberté complète d'usage des revenus financiers du lait.

Individuellement, les productrices semblent intéressées par le projet de structuration de la filière. Elles voient deux avantages à cette structuration: elles n'auront plus à baratter le lait que les hommes auront collecté frais et pourront vendre leur production plus cher. Leur travail sera allégé mais leurs produits seront moins diversifiés. Le mari s'autorisera un droit de regard sur l'augmentation des revenus.

33 Ainsi, une certaine constante dans la répartition des tâches rend compte de l'oppression des femmes qui se traduit dans le domaine technique et dans la maîtrise, politique et symbolique ${ }^{17}$. Apparaît la notion de hiérarchisation qui est essentielle pour analyser non seulement les inégalités de fait, mais aussi leur perpétuation.

Les limites de l'approche selon le genre

On saisit les mieux la hiérarchisation des rapports sociaux de genre lorsqu'on les saisit au regard des enjeux économiques dans lesquels ils s'inscrivent. Aussi, cette étude ne serait pas complète si elle ne tenait pas compte des autres protagonistes opérant dans la sous-filière du commerce du lait. Ce sont des femmes appelées collectrices qui pratiquent, habituellement, le commerce du lait caillé dans les agglomérations rurales périphériques de Ndjaména, pour le revendre à la capitale. Ce sont désormais des hommes qui collectent le lait frais à mobylette sur les mêmes axes goudronnés où commercent les femmes.

Des actrices et des acteurs en concurrence?

A la typologie selon les activités commerciales (grossistes-intermédiaires-revendeuses-détaillantes) réalisée par la mission d'identification de 1997, nous avons superposé une typologie plus sociologique qui distingue les femmes collectrices rurales (un tiers des enquêtées) des femmes collectrices urbaines (deux tiers).

Les collectrices rurales sont assez jeunes et collectent le lait caillé depuis quatre ans en moyenne. Elles vivent dans les villages de la périphérie de Ndjaména dans la préfecture $\mathrm{du}$ Chari-Baguirmi. La plupart sont des femmes arabes du Chari-Baguirmi. Contrairement aux femmes d'éleveurs, les collectrices rurales vivent une assez grande instabilité matrimoniale ${ }^{18}$. Nous les avons rencontrées sur les marchés les plus importants de la préfecture jusqu'aux rives du lac $(150 \mathrm{~km})$. Les plus grosses quantités collectées y sont le fait des collectrices grossistes qui revendent à Ndjaména : entre 6 et 25 bidons de 40 litres collectés par semaine en saison sèche. Pour réduire les frais de transport, certaines se groupent et envoient deux d'entre elles à tour de rôle à la capitale chaque semaine. En saison des pluies elles réduisent, ou cessent leurs déplacements pour s'adonner au travail agricole. Les détaillantes se déplacent sur de 
plus faibles distances et transportent des quantités moindres (120 litres au maximum). Ces collectrices intermédiaires et détaillantes revendent dans les villages et sur les marchés avoisinants en dehors des jours de marché.

A Ndjaména, les collectrices qui, en général, ne peuvent, faute de temps, rester en ville pour détailler elles-mêmes leur collecte, doivent parfois "casser" les prix en revendant aux grossistes leur bidon de 40 litres pour 4500 FCFA au lieu de 5000, voire 5500 FCFA. Elles sont "soumises" au "quasi-monopole" des collectrices grossistes urbaines qui possèdent un magasin d'entreposage sur les marchés. Ces dernières, quant à elles, sont d'origine ethnique diverse et représentent le melting-pot propre aux grandes villes. La plupart d'entre elles provient cependant des groupes du nord du Tchad liés au commerce et/ou à l'élevage. Toutes vivent à la capitale depuis fort longtemps.

Ces femmes sont généralement plus âgées que les collectrices rurales. Nous pouvons y distinguer deux générations : la première est arrivée après la sécheresse de 1973 qui a décimé les troupeaux; la seconde après la sécheresse de 1983-1984. Les plus récentes dans le métier sont arrivées après la prise du pouvoir par l'actuel président Idriss Déby. Dans la majorité des cas "c'est la pauvreté qui les a amenées en ville" et plus de la moitié d'entre elles étaient à l'origine des talanés, c'est-à-dire des femmes d'éleveurs nomades vendant leur lait frais et caillé à domicile. Leur situation sociale et matrimoniale est précaire" ${ }^{19}$. Pour la plupart veuves ou divorcées, elles vivent avec un frère, un fils ou une fille et son gendre. Souvent ces "vieilles mamans" sont les deuxièmes personnes, après le gendre ou le fils, à subvenir aux besoins de la famille. Pour surmonter les difficultés économiques que connaissent nombre de familles urbaines et en face desquelles ne résisteraient pas les liens sociaux (Locoh 1997), ces femmes collectrices, parfois très âgées, n'entendent pas être une charge supplémentaire. Les gains provenant du commerce de lait caillé apparaissent donc comme une part essentielle à la survie de ces familles urbaines.

Les femmes collectrices urbaines vendent individuellement en moyenne à Ndjaména 12,7 bidons de 40 litres par semaine, en saison sèche. En saison des pluies, les stratégies divergent et, si la majorité d'entre elles doublent leurs quantités collectées, d'autres préfèrent réduire leurs déplacements ou bien cesser la collecte pendant deux à trois mois. Les prix sont moins intéressants, les déplacements plus difficiles. En totalisant les coûts de transport et le loyer de leur magasin, nous avons calculé une marge bénéficiaire individuelle moyenne de 14650 FCFA par semaine, ce qui équivaut en arrondissant à 2000 FCFA par jour.

Du côté des hommes, on observe un développement de l'activité de collecte de lait frais à mobylette, les plus récemment arrivés dans ce commerce se contentant d'une bicyclette. La moitié d'entre eux s'est regroupée récemment (1997) au sein de cinq associations. Il s'agit d'hommes de moins de 40 ans, agro-éleveurs pour la plupart. Leur pratique de collecte remonte à quatre ans en moyenne, sept ans pour les plus anciens. Nombre d'entre eux étaient des commerçants auparavant, profitant d'un commerce de contrebande grâce à la proximité des frontières camerounaise et nigériane. Depuis l'avènement du président Idriss Déby, les douaniers se montrent d'une "efficacité" sans précédent qui les a obligés à se rabattre sur la collecte du lait, moins lucrative que la contrebande de sucre ou d'essence, mais licite.

41 Les hommes enquêtés collectent en moyenne dans quatre à six villages auxquels s'ajoutent quelques campements d'éleveurs nomades (ferrick) en saison sèche. Ceci équivaut à la production de trente à soixante femmes. Les distances parcourues sont 
assez élevées et il n'est pas rare qu'elles atteignent $160 \mathrm{~km}$ (aller et retour) ${ }^{20}$. En saison des pluies, deux à trois villages saturent leur capacité de transport. Arrivés en ville, tous les collecteurs revendent à des points de vente "lait pur vache". L'enseigne est visible, uniformisée et provient, semble-t-il, de produits importés. Ces points de vente sont de petites boutiques de commerce général qui, parfois, réservent un emplacement propre à la consommation de jus de fruits et de rayib, sorte de yahourt à base de lait fermenté entier. Cette sous-filière est déjà relativement structurée et chaque collecteur dispose de plusieurs de ces lieux de livraison implicitement attitrés.

Confrontés à des charges récurrentes, telles que les frais d'électricité, de conservation, ou à des problèmes inhérents au marasme économique et à la faible solvabilité de la clientèle, les boutiquiers diffèrent le paiement aux revendeurs de lait. Il en résulte des tensions mais l'intérêt lucratif de la collecte du lait frais reste suffisant pour motiver sa poursuite. Le gain net moyen calculé pour chaque collecteur, en incluant les frais de carburant (mais non l'entretien de la mobylette), s'élèverait à 7500 FCFA par jour en saison sèche et approcherait le double en saison des pluies.

Les enjeux de la concurrence

L'engagement des acteurs masculins dans la sous-filière du lait frais a fait perdre une part de marché importante à la sous-filière du lait caillé. Néanmoins celle-ci ne peut disparaître totalement en raison de l'habitude alimentaire des populations sahéliennes : le lait caillé, notamment en période de Ramadan reste très prisé et permet de conserver le lait de façon très efficace. Les femmes collectrices sont peu organisées pour lutter contre une perte de marché. Les contraintes sociologiques se résument à leur besoin d'autonomie financière face au désengagement des hommes dans la sphère familiale et à la mercantilisation de l'économie en milieu urbain. Le capital financier des femmes demeure modeste et ne les invite pas à diversifier leur produit ni à lutter contre la concurrence. De plus, par manque de familiarité et d'information, elles n'ont que très rarement recours aux institutions d'aide extérieures. Elles n'expriment pas leurs besoins de structuration ou de structuration de leur sous-filière. Leur pratique individuelle est encore ce qui les insécurise le moins. Elles se trouvent contraintes, par manque d'information quant aux enjeux sur le marché laitier, de limiter la concurrence entre elles, notamment dans le rapport entre collectrices urbaines et rurales, sans s'autoriser à tenir compte du rapport de force introduit par l'activité des hommes. Les femmes d'éleveurs ne maîtrisent pas l'outil que représentent le groupement et l'action collective organisée et ne s'expriment pas quant aux conditions nouvelles que crée l'activité des hommes. "Les femmes ne défient pas ouvertement leurs conditions de subordination" (Cloutier et Sy 1993).

Quant aux hommes, même fortement imprégnés d'individualisme et d'esprit d'indépendance, ils formulent des revendications explicites dans le domaine technique (aide financière pour réparer les mobylettes, formation pour la fabrication du fromage) et dans la dynamique organisationnelle (centralisation des achats et transport des compléments alimentaires pour le bétail ; groupement en association et contrôle des collecteurs). Ces revendications sont légitimes dans la mesure où ils détiennent les moyens de production, les instruments de travail et la reconnaissance de leur droit d'usage et maîtrisent, en outre, les procédés de négociation et les réseaux d'interlocuteurs adéquats, tels que les opérateurs du développement et les bailleurs de fonds.

Des "sujets-agis", ou des limites de l'analyse selon le genre 

luttes. Il leur réserve un espace limité, féminin. Et les termes de référence de l'étude selon le genre nous rappellent cette distinction à la fois spatiale et politique des hommes et des femmes, de leurs activités et de leurs positions dans les enjeux de la filière. On caractérise alors leurs pratiques par des savoir-faire relevant du traditionalisme plutôt que d'usages anciens (la tradition est idéologiquement utilisée, non historiquement expliquée), légitimant d'autant et réitérant leur inscription dans un informel plus spécifique des femmes. La formation des femmes est perçue comme l'était celle des femmes du monde ouvrier en France : "l'acquisition de leur savoir-faire se faisant hors des canaux institutionnels de qualification, toujours en référence à la sphère du privé, (leur) apparaît comme une acquisition individuelle et non collective" (Kergoat $1984: 211$ ). femmes sont sujets de l'analyse autant que de leur histoire. Mais comme nous le dit très bien N.C. Mathieu, elles sont des "sujets-agis". Les contraintes sociales n'autorisent le jeu de la concurrence que dans la sphère féminine où les femmes ont été reléguées par l'analyse avant de l'être par la pratique du développement. La demande d'une étude de genre en termes descriptifs, voire prescriptifs mais non analytiques (Planté 1991) les a renvoyées à une spécificité féminine. L'étude des rapports sociaux de concurrence, qui ne sont pas l'apanage d'un sexe, échappe à ce type d'analyse.

47 Passer à la réalité dans sa dimension globale, c'est à dire aborder le corps social entier comme marqué par les rapports de genre, i.e., rapports socio-sexués hiérarchisés (envisager que les femmes rivalisent avec des moyens équivalents à ceux des hommes, avec eux), élargit la lutte à l'ensemble du champ du politique.

48 Lorsque les études avant-projet font du genre un habit neuf pour une vieille notion de spécificité féminine, la frontière politique du projet est tracée. Sa mise en application "sur le terrain" révèle une ambiguïté qui n'est pas sans lien avec la "légitimité institutionnelle" (Scott 1988) qui neutralise, par l'a-politisme, toute revendication de changement.

\section{Conclusion}

49 L'instabilité de la traduction et de la définition du concept de genre s'articule sur plusieurs niveaux. D'une part, la traduction en français du terme gender crée une confusion pour des raisons historiques et culturelles tant au niveau de son emploi grammatical (où genre $=$ sexe) que dans son utilisation dans d'autres sciences, en particulier en sciences naturelles où le genre est un outil taxinomique qui ne retient pas le sexe (Planté 1991). Le risque de renaturaliser les rapports sociaux de genre en résulte. Au vrai, cette confusion, "loin de constituer un pur problème terminologique, est éminemment révélatrice du statut problématique des femmes au regard de la notion d'humanité, dans de nombreux discours normatifs et scientifiques du XIXe siècle : à la fois dedans et dehors, composantes nécessaires, cas limites et exceptions" (Planté 1991 : 55).

Cette imprécision laisse libre l'espace d'imposition des catégories dominantes, i.e. masculines, sur les pratiques sociales réelles. Même en tant que sujets dans la réalité d'un contexte de projet où la hiérarchisation est éclairée à travers des enjeux, les femmes restent des "sujets-agis". Autrement dit, elles ont une conscience qui n'est pas . identique à celle des dominants" (Mathieu 1985). Ne pas voir cela, signifie adopter le point de vue hégélien qui conduit à " un éloignement conséquent d'une analyse 
matérialiste de la conscience" (ibid.) et invite à un idéalisme absolu ${ }^{21}$ (Godelier 1977, cité par Mathieu 1985).

51 Confondre le genre et le sexe féminin dans les études participe de cet idéalisme absolu qui permet d'éviter l'analyse des rapports sociaux réels face aux enjeux et aux ressources. Le genre contient, en effet, une dimension idéologique qui participe de sa construction culturelle qui échappe à l'analyse de classes impliquant la seule causalité matérialiste. "Tandis que la catégorie de "classe" repose sur la théorie complexe de Marx et sur ses développements ultérieurs de la détermination économique et du changement historique, celles de "race" et de "genre" ne véhiculent pas de pareilles associations. (...) Il n'y a pas ce type de clarté ou de cohérence ni pour la catégorie de race ni pour celle du genre. Dans le cas du genre, son usage a impliqué un éventail aussi bien de positions théoriques que de références descriptives des rapports entre les sexes" (Scott 1988 : 127). S'"il y a toujours une place pour une lutte cognitive à propos du sens des choses du monde ", dans l'espace étroit que constitue "la correspondance entre les réalités ou les processus du monde naturel et les principes de vision et de division qui leur sont appliqués" (Bourdieu 1998 : 20), cette place est-elle pour autant "une possibilité de résistance" offerte aux dominés "contre l'effet d'imposition symbolique", comme le suggère Bourdieu? Ou n'est-elle pas, dans notre cas, le lieu même où se renforce cet "effet d'imposition symbolique" des dominants?

Autrement dit, "l'introduction du genre dans les sciences humaines répond à une politique : présenter les traits de genre comme symboliques ou arbitraires en laissant au sexe anatomique le rôle de réel incontournable. Les précédents de ce type d'opérations (telle la tentative de remplacement de "race" par "ethnie") indiquent pour le moins leur ambiguïté" (Guillaumin, citée par Mathieu 1991b: 79). L'usage actuel du concept de genre dans les études avant projet autorise, en même temps qu'il paralyse, les changements structurels qu'impliquerait son potentiel heuristique.

\section{BIBLIOGRAPHIE}

Bisilliat J., 1997, Face aux changements. Les femmes du Sud, Paris, L'Harmattan.

Bonfiglioli A.M., 1981, Le baton et la houe. Une introduction à l'agro-pastoralisme du Sahel tchadien, Ndjaména, Documentation du Cefod.

Bonfiglioli A.M., 1990, "Pastoralisme, agro-pastoralisme et retour : itinéraires sahéliens", Cahiers des Sciences Humaines, XXVI (1-2) : 255-266.

Bourdieu P., 1998, La domination masculine, Paris, Editions du Seuil.

Bourzat D., Souvenir Zafindrajoana P., Dubois P., 1998, "Etude du marché du lait et des produits laitiers dans le Bassin de collecte de Ndjaména. Impact de la dévaluation sur la filière lait", Communication à l'Atelier-Recherche : Marchés urbains et développement laitier en Afrique Subsaharienne, Cirad, Montpellier. 
Boutinot L., 1995, "Du discours à la réalité. Les mouvements féministes : de la défensive à l'offensive", Réseau Femmes et Développement, Bulletin thématique, Montreuil.

Bruggeman H., 1991, Femmes pastorales et gestion du bétail : exemples tirés du nord de l'Ouganda et du centre du Tchad, Ndjaména, Rapport de mission, OXFAM.

CERM (Centre d'Etudes et de Recherches Marxistes), 1978, La condition féminine, Paris, Editions Sociales.

Charmes J., 1997, "Plus visibles mais toujours sous-estimées : l'activité économique des femmes en Afrique", in Bisilliat J. (éd.), Face aux changements, les femmes du sud, Paris, L'Harmattan : 47-58.

Cloutier L., Djibrine Sy A., 1993, Ndjaménoise du secteur informel, Ndjaména, Bulletin du Cefod.

Combes D., Daune-Richard A.M., Devreux A.M., 1991, "Mais à quoi sert une épistémologie des rapports sociaux de sexe ?" in Hurtig M.C. et al. (éds) Sexe et genre. De la hiérarchie entre les sexes, Paris, CNRS : 59-68.

DAWN (Development Alternatives with women for a new era), 1992, Femmes du sud. Autres voix pour le XXI ${ }^{e}$ siècle, Paris, Editions Côté Femmes.

Delphy C., 1991, "Penser le genre : Quels problèmes ?", in Hurtig M.C. et al. (éds) Sexe et genre. De la hiérarchie entre les sexes, Paris, CNRS : 89-101.

Dupire M., 1970, L'organisation sociale des Peul, Paris, Plon.

Gauthier J., Boutinot L., Koussou M., 1998, Etude complémentaire sur la filière lait au Tchad, Montpellier, Rapport Cirad-Emvt, $n^{\circ}$ 98-030.

Godelier M., 1977, Horizon, trajets marxistes en anthropologie, Nouvelle Edition, Paris, Maspero.

Hurtig M.C., Kail M., Rouch H. (éds), 1991, Sexe et genre. De la hiérarchie entre les sexes, Paris, CNRS.

Kergoat D., 1984, Plaidoyer pour une sociologie des rapports sociaux.., in Collectif (éd.), Le sexe du travail. Structures familiales et système productif, Grenoble, Presses Universitaires de Grenoble : 207-220.

Koussou Miang-Ondanag, 1998, L'approvisionnement de la ville de Ndjaména en produits laitiers, Ndjaména, LRVZ.

Lagrave R.M., Caniou J., 1987, Celles de la terre. Agricultrices, l'invention politique d'un métier, Paris, EHESS.

Lyklema a Nijeholt G., 1987, The fallacy of integration : the UN strategy of integrating women revisited.., Netherlands Review of Development Studies, I.

Locoh T., Hesseling, G., 1997, "Femmes, pouvoir et sociétés", Politique Africaine, 65 : 3-20.

Mathieu N.C. (éd.), 1985, L'arraisonnement des femmes. Essais en anthropologie des sexes, Cahiers de l'Homme, Paris, EHESS.

Mathieu N.C., 1991a, "Tendances actuelles de la recherche en anthropologie des sexes", in Mathieu, N.C. (éd.), Les relations hommes je mm es dans le bassin du Lac Tchad, Paris, Editions ORSTOM.

Mathieu, N.C., 1991b, "Les transgressions du sexe et du genre à la lumière de données ethnographiques", in Hurtig M.C. et al. Sexe et genre. De la hiérarchie entre les sexes, Paris, CNRS : 69-80. Michel A., 1979, Le féminisme, Paris, PUF.

Planté E., 1991, "La confusion des genres", in Hurtig M.E. et al., Sexe et genre. De la hiérarchie entre les sexes, Paris, CNRS : 51-57. 
Scott J., 1988, "Genre : une catégorie d'analyse historique", Les Cahiers du GRIF, Editions Tierce, (37-38) : 124-153.

Spencer B., 1999, "La femme sans sexualité et l'homme irresponsable", Actes de la Recherche en Sciences Sociales, $128: 29-33$.

Souvenir Zafindrajoana P., Gauthier J., Bouchel D., Koussou M., 1997, Mission d'identification d'un projet visant à l'amélioration de l'approvisionnement laitier de Ndjaména, Montpellier, Rapport Cirad-Emvt, $n^{\circ}$ 97-022.

Sullerot E., 1978, Le fait féminin, qu'est-ce qu'une femme ?, Paris, Fayard.

Vatin F., 1990, L'industrie laitière. Essai d'histoire économique, Paris, l'Harmattan.

\section{NOTES}

1.... un concept hérité du monde anglo-saxon mais désormais bien présent en France...

2.Le cheptel est estimé par la direction de l'élevage à 5,5 millions de têtes de bovins (dont les différentes races sont les zébu arabe, bororo et le taurin Kouri) 2,4 M d'ovins, 4,8 M de caprins et 560000 dromadaires en 1997.

3.Centre de Modernisation et de Production animale (financements suisses) en 1967 et le projet de création d'une laiterie industrielle à Farcha, à la périphérie de Ndjaména en 1975.

4.Notamment à travers un programme financé par la Communauté européenne.

5.En saison des pluies il est difficile "d'évaluer si la population actuelle de Ndjaména absorbe les surplus". "Mais la période de réelle surproduction ne semble être répartie que sur trois mois. La chute des prix pendant les mois de juillet, août, septembre indique cependant une demande pleinement satisfaite pendant celte période". (Mission d'identification, p. 57).

6.Les problèmes hydrauliques ne sont pas évoqués dans la mesure où ils sont pris en charge par d'autres projets de développement.

7.Ces calculs sont à prendre avec précaution. Par exemple. le fait d'annoncer qu'il y aurait environ 30000 tonnes/an de tourteaux disponibles en considérant qu'il n'y ait que les GDS - groupement de défense sanitaire - de la zone soudanienne qui en achètent pour nourrir les bovins de tractation et pour la fumure; et le fait de considérer qu'avec une alimentation complémentée les vaches produiraient quotidiennement deux fois plus de lait soit 1.6 litres au lieu de 0.8 litre. 8. La SOMAFA est une société de fabrication alimentaire à caractère privé spécialisée dans la production de jus de fruits. Elle cherche à développer une activité de laiterie.

9.Cf. Gauthier, Boutinot, Koussou (1998).

10.Bonfiglioli (1990).

11.Le recueil des données quantitatives concernant le bétail est toujours délicat. Avec réserves, nous avons obtenu les chiffres suivants : $43 \%$ des femmes possèdent des vaches ou chamelles, $19 \%$ des vaches et 
des petits ruminants, $19 \%$ uniquement des petits ruminants, $19 \%$ aucun animal. L'avoir bovin et/ou camelin s'élèverait à environ 7 à 8 têtes par femme, les petits ruminants à 9 ou JO têtes. Cf. Gauthier, Boutinot, Koussou (1998).

12.Seules deux femmes (sur 11) nous ont dit avoir reçu un petit ruminant sous forme de dot ou d'héritage.

$13.70 \mathrm{~km}$ représentent 200 FCFA de variation en saison sèche et 50 à 100 FCFA, en saison pluvieuse.

14. Le coro équivaut à 2 litres. Par exemple un cob, sur le marché de Linia en mars 1998, vaut 75 FCFA. Le coro sera à 187,5 FCFA. A Ndjaména, le cob est vendu à 150 FCFA et le coro revient à 375 FCFA. Par ailleurs, 100 FCFA $=1 \mathrm{FF}$.

15.Il convient de noter que l'équivalent du SMIG en milieu urbain à Ndjaména (petits fonctionnaires, techniciens du secteur privé industriel) est environ de 25000 FCF A par mois.

16.Il existe, en effet, un serment sur le lait. Entretien avec M. Dupire. 17.Cf. Tabet P., "Les mains, les outils, les armes". L'Homme XIX (3-4) 1979. citée par N.E. Mathieu, op. cit. p. 27.

18.Cf. Rappon de mission op. cit.

$19.39 \%$ de notre échantillon sont veuves, $28 \%$ divorcées, $22 \%$ vivent avec un mari inactif et $11 \%$ vivent avec un mari actif. Échantillon de 19 femmes sur environ 200.

20.Ces performances se réalisaient par voie de chemin de fer au XIXe siècle pour l'approvisionnement laitier de la ville de Paris. Cf. F. Vatin, L'industrie du lait. Essai d'histoire économique, Paris, L'Harmattan, 1990, p 60.

21.Les mots sont soulignés par l'auteur, M. Godelier.

\section{AUTEUR}

\section{LAURENCE BOUTINOT}

Sociologue, Cirad. Forêt. 